\title{
SUFFICIENT CONDITIONS FOR THE ISOPERIMETRIC PROBLEM OF BOLZA IN THE CALCULUS OF VARIATIONS
}

\author{
BY \\ MAGNUS R. HESTENES
}

1. Introduction. The present paper is the last of a sequence of three papers on the isoperimetric problem of Bolza. The first paper was concerned with certain properties of the Weierstrass $E$-function. The second paper dealt with the Theorem of Lindeberg and its consequences. The present paper is concerned with a proof of a sufficiency theorem for a strong relative minimum conjectured by McShane $\left.{ }^{1}\right)$. The corresponding theorem for a weak relative minimum was established by McShane and his method was used by Myers $\left({ }^{2}\right)$ to obtain sufficient conditions for a semistrong relative minimum for the nonparametric problem of Lagrange. Moreover Myers showed that these conditions were sufficient for a strong relative minimum when the integrand of the integral $I(C)$ to be minimized had certain special properties. Using an extension of the methods devised by McShane and Myers we establish a sufficiency theorem for a proper strong relative minimum in which an estimate is given of the difference $I(C)-I\left(C_{0}\right)$ for the arcs $C$ under consideration. This added feature enables one to obtain analogues of the theorem of Osgood as a corollary to our sufficiency theorem. Another interesting feature of the method here used is that it is applicable even when isoperimetric conditions are present. The methods used heretofore for problems in which isoperimetric conditions are present usually involved a transformation of the problem or involved the theory of broken extremals. Throughout the paper it was found that many of the ideas which play a prominent role in the field theory are also useful in the proofs here given.

2. Hypotheses and preliminary definitions. The problem with which we are concerned is that of finding in a class of arcs

$C: \quad a^{h}, \quad y^{i}(t) \quad\left(t^{1} \leqq t \leqq t^{2} ; h=1, \cdots, r ; i=0,1, \cdots, n\right)$ satisfying conditions of the form

$$
\begin{gathered}
\phi^{\beta}(a, y, \dot{y})=0 \quad(\beta=1, \cdots, m<n), \\
y^{i}\left(t^{1}\right)=T^{i 1}(a), \quad y^{i}\left(t^{2}\right)=T^{i 2}(a),
\end{gathered}
$$

Presented to the Society, September 13, 1943; received by the editors November 9, 1945.

(1) E. J. McShane, Sufficient conditions for a weak relative minimum in the problem of Bolza, Trans. Amer. Math. Soc. vol. 52 (1942) pp. 344-379. See also M. J. Cox, On necessary conditions for relative minima, Amer. J. Math. vol. 66 (1944) pp. 170-198.

(2) F. G. Myers, Sufficient conditions for the problem of Lagrange, Duke Math. J. vol. 10 (1943) pp. 73-97. 


$$
I^{\sigma}(C)=g^{\sigma}(a)+\int_{C} f^{\sigma}(a, y, \dot{y}) d t=0
$$

one which minimizes the function

$$
I(C)=g(a)+\int_{C} f(a, y, \dot{y}) d t .
$$

The notations and terminology described in the first paper $\left(^{3}\right)$ will be used throughout. In particular arcs will be written in the vector form

$C:$

$$
a, \quad y(t)
$$$$
\left(t^{1} \leqq t \leqq t^{2}\right)
$$

and an admissible arc is a rectifiable arc having an absolutely continuous representation $C$ such that for almost all values of $t$ on $t^{1} t^{2}$ the element $[a, y(t), \dot{y}(t)]$ is admissible and satisfies the equations (2.1), (2.2), and (2.3).

We center our attention on a particular admissible arc $C_{0}$ of class $C^{\prime \prime}$ with a representation

$C_{0}$ :

$$
a_{0}, \quad y_{0}(t)
$$$$
\left(t^{1} \leqq t \leqq t^{2}\right)
$$

of class $C^{\prime \prime}$ such that $\dot{y}_{0}(t) \neq 0$. It is assumed that $C_{0}$ does not intersect itself and that the matrix $\left\|\phi_{p^{i}}\right\|$ has rank $m$ on $C_{0}$. By a variation $\gamma$ will be meant a set of constants and absolutely continuous functions

$$
\gamma: \quad \alpha^{h}, \quad \eta^{i}(t) \quad\left(t^{1} \leqq t \leqq t^{2} ; h=1, \cdots, r ; i=0,1, \cdots, n\right)
$$

whose derivatives $\dot{\eta}^{i}(t)$ are integrable together with their squares. Here too we shall use the vector notation

$\gamma$ :

$$
\alpha, \quad \eta(t)
$$$$
\left(t^{1} \leqq t \leqq t^{2}\right)
$$

A variation $\gamma$ will be said to be differentially admissible if it satisfies the differential equations

$$
\Phi^{\beta}(t, \alpha, \eta, \dot{\eta})=\phi_{a h \alpha}^{\beta}{ }^{h}+\phi_{v^{i} \eta^{i}}^{\beta}(t)+\phi_{p^{i} \dot{\eta}^{i}}^{\beta}(t)=0
$$

for almost all values of $t$ on $t^{1} \leqq t \leqq t^{2}$. In this equation the arguments in the derivatives of $\phi^{\beta}$ are $\left[a_{0}, y_{0}(t), \dot{y}_{0}(t)\right]$. By an admissible variation $\gamma$ will be meant one that is differentially admissible and satisfies the conditions

$$
\begin{gathered}
\eta^{i}\left(t^{1}\right)=T_{h}^{i 1} \alpha^{h}, \quad \eta^{i}\left(t^{2}\right) \doteq T_{h}^{i 2}{ }^{h}, \\
I_{1}^{\sigma}(\gamma)=g_{h}^{\sigma} \alpha^{h}+\int_{t_{1}}^{t_{2}} \psi^{\sigma}(t, \alpha, \eta, \dot{\eta}) d t=0,
\end{gathered}
$$

where $T_{h}^{i 1}, T_{h}^{i 2}, g_{h}^{\sigma}$ are the derivatives of $T^{i 1}(a), T^{i 2}(a), g^{\sigma}(a)$ with respect to $a^{h}$ at $a=a_{0}$ and

(3) M. R. Hestenes, The Weierstrass E-function in the calculus of variations, Trans. Amer. Math. Soc. vol. 60 (1946) pp. 51-71. This paper will be referred to as the "first paper." 


$$
\psi^{\sigma}(t, \alpha, \eta, \pi)=f_{a^{h} \alpha}^{\sigma}{ }^{h}+f_{y^{i} \eta^{i}}^{*}+f_{p^{i} \pi}^{\sigma},
$$

the arguments in the derivatives of $f^{\sigma}$ being $\left[a_{0}, y_{0}(t), \dot{y}_{0}(t)\right]$. An admissible variation $\gamma$ will be said to be essentially null if it is of the form

$$
\alpha^{h}=0, \quad \eta^{i}(t)=\rho(t) \dot{y}_{0}^{i}(t)
$$

In this case $\rho\left(t^{1}\right)=\rho\left(t^{2}\right)=\eta^{i}\left(t^{1}\right)=\eta^{i}\left(t^{2}\right)=0$ by virtue of the condition (2.6). Consider now a set of multipliers

$$
l^{0} \geqq 0, \quad l^{\sigma}, \quad m^{\beta}(a, y)
$$

of class $C^{\prime}$ on a neighborhood of $C_{0}$. The multipliers $l^{0}, l^{\sigma}$ are constants. Set

$$
F(a, y, p, l, m)=l^{0} f+l^{\sigma} f^{\sigma}+m^{\beta} \phi^{\beta}, \quad G(a, l)=l^{0} g+l^{\sigma} g^{\sigma} .
$$

The function

$$
J(C)=G(a, l)+\int_{C} F[a, y, \dot{y}, l, m(a, y)] d t
$$

has as its first variation along $C_{0}$ the function

$$
J_{1}(\gamma)=G_{h} \alpha^{h}+\int_{i_{1}}^{t_{2}}\left[F_{a^{h} \alpha^{h}}+F_{y^{i} \eta^{i}}+F_{p^{i} \eta^{i}}\right] d t .
$$

In this expression the derivatives of $G$ and $F$ are to be evaluated along $C_{0}$. Here $G_{h}$ is the derivative of $G$ with respect to $a^{h}$. It is well known that the condition

$$
J_{1}(\gamma)=0
$$

holds for every variation $\gamma$ satisfying the end conditions (2.6) if and only if $C_{0}$ satisfies the Euler-Lagrange equations

$$
\frac{d}{d t} F_{p^{i}}=F_{y^{i}}, \quad \phi^{\beta}=0
$$

and the transversality conditions

$$
G_{h}+F_{p^{i}}\left(t^{2}\right) T_{h}^{i 2}-F_{p i}\left(t^{1}\right) T_{h}^{i 1}+\int_{t_{1}}^{t_{2}} F_{a d} d t=0 .
$$

Here $F_{p^{i}}\left(t^{1}\right)$ and $F_{p^{i}}\left(t^{2}\right)$ denote the values of $F_{p^{i}}$ at the initial and final end points of $C_{0}$.

In order to define the second variation $J_{2}(\gamma)$ of $J(C)$ along $C_{0}$ we define $2 \Omega$ by the formula

$$
\begin{aligned}
& 2 \Omega(t, \alpha, \eta, \pi)=F_{y^{i} y^{i} \eta^{i} \eta^{j}}+2 F_{y^{i} p^{i} \eta^{i} \pi^{j}}+F_{p^{i} p^{i} \pi^{i} \pi^{j}}
\end{aligned}
$$

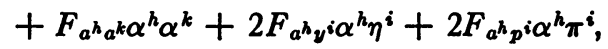


where the derivatives of $F$ are evaluated along $C_{0}$. We also set

$$
b_{h k}=G_{h k}+F_{p^{i}}\left(t^{2}\right) T_{h k}^{i 2}-F_{p^{i}}\left(t^{1}\right) T_{h k}^{i 1}
$$

where the subscripts $h, k$ on $G, T^{i 1}, T^{i 2}$ denote derivatives with respect to $\alpha^{h}, \alpha^{k}$ evaluated at $a=a_{0}$. As before $F_{p^{i}}\left(t^{1}\right)$ and $F_{p^{i}}\left(t^{2}\right)$ are the values of $F_{p^{i}}$ at the end points of $C_{0}$. The function

$$
J_{2}(\gamma)=b_{h k} \alpha^{h} \alpha^{k}+\int_{t^{1}}^{t^{2}} 2 \Omega(t, \alpha, \eta, \dot{\eta}) d t
$$

is called the second variation of $J(C)$ along $C_{0}$.

3. The conditions $A, B, A^{\prime}$ and $B^{\prime}$. Following the notations used in the earlier papers we shall denote by $\mathfrak{D}$ the class of all differentially admissible elements $(a, y, p)$, that is, all admissible elements $(a, y, p)$ such that $\phi^{\beta}(a, y, p)=0$. Associated with each admissible function $H(a, y, p)$ we have the Weierstrass $E$-function

$$
E_{H}(a, y, p, q)=H(a, y, q)-q^{i} H_{p^{i}}(a, y, p) .
$$

Let $F(a, y, p)$ and $H(a, y, p)$ be admissible. The function $H(a, y, p)$ will be said to be $E$-dominated by $F(a, y, p)$ near $C_{0}$ on $\mathfrak{D}$ if there is a constant $b>0$ and a neighborhood $\mathfrak{D}_{0}$ of $C_{0}$ relative to $\mathfrak{D}$ such that the inequality

$$
\left|E_{H}(a, y, p, q)\right| \leqq b E_{F}(a, y, p, q)
$$

holds whenever $(a, y, p)$ is in $\mathfrak{D}_{0}$ and $(a, y, q)$ is in $\mathfrak{D}$.

The arc $C_{0}$ will be said to satisfy the condition $\mathrm{A}$ with a set of multipliers

$$
l^{0} \geqq 0, \quad l^{\sigma}, \quad m^{\beta}(a, y)
$$

if the following conditions hold: the functions $m^{\beta}(a, y)$ are of class $C^{\prime}$ on a neighborhood of $C_{0}$; the Euler-Lagrange equations (2.14) and transversality conditions (2.15) hold with $F(a, y, p, l, m(a, y))$ and $G(a, l)$ defined by (2.11); the function $H \equiv 0$ is $E$-dominated by $F(a, y, p, l, m(a, y))$ near $C_{0}$ on $\mathfrak{D}$. If in addition the functions $L(p)=|p|$ and $f^{\sigma}(a, y, p)(\sigma=1, \cdots, s)$ are $E$-dominated by $F(a, y, p, l, m(a, y))$ near $C_{0}$ on $\mathfrak{D}$, then $C_{0}$ will be said to satisfy the condition $\mathrm{A}^{\prime}$ with the set of multipliers (3.3). In this event the functions $\phi^{\beta}(a, y, p)(\beta=1, \cdots, m)$ are also $E$-dominated by $F(a, y, p, l, m(a, y))$ near $C_{0}$ on $\mathfrak{D}$, as was seen in Theorem 4.3 of the first paper.

The arc $C_{0}$ will be said to satisfy the condition B if for every admissible variation $\gamma$ that is not essentially null, there is a set of multipliers (3.3) with which $C_{0}$ satisfies condition A such that the inequality $J_{2}(\gamma)>0$ holds, where $J_{2}(\gamma)$ is the second variation (2.18) of the function $J(C)$ determined by these multipliers. If in condition $B$ we require that the multipliers (3.3) satisfy the condition $\mathrm{A}^{\prime}$ with $C_{0}$ then $C_{0}$ will be said to satisfy the condition $\mathrm{B}^{\prime}$.

LEMMA 3.1. If $C_{0}$ satisfies condition $\mathrm{B}$ and there is a set of multipliers (3.3) 
with which $C_{0}$ satisfies condition $\mathrm{A}^{\prime}$, then $C_{0}$ satisfies condition $\mathrm{B}^{\prime}$. Conversely if $C_{0}$ satisfies condition $\mathrm{B}^{\prime}$, then it satisfies condition $\mathrm{B}$ and there is a set of multipliers (3.3) with which it satisfies condition $\mathrm{A}^{\prime}$.

For suppose $C_{0}$ satisfies condition $\mathrm{A}^{\prime}$ with the multipliers $\bar{l}^{0}, l^{\beta}, \bar{m}^{\beta}(a, y)$ and let $\gamma$ be an admissible variation that is nonessentially null. If $C_{0}$ satisfies the condition B there is a set of multipliers (3.3) with which $C_{0}$ satisfies condition A such that $J_{2}(\gamma)>0$. For a sufficiently small positive constant $b$ the arc $C_{0}$ will satisfy condition $A^{\prime}$ with the multipliers

$$
l^{0}+b \bar{l}^{0}, \quad l^{\sigma}+b \bar{l}^{\sigma}, \quad m^{\beta}(a, y)+b \bar{m}^{\beta}(a, y)
$$

and the second variation of the function $J(C)$ determined by these multipliers remains positive on $\gamma$. Hence $C_{0}$ satisfies condition B' $^{\prime}$. The converse follows from the fact that there exists an admissible variation $\gamma$ that is not essentially null $\left({ }^{4}\right)$.

LEMMA 3.2. If $C_{0}$ satisfies condition $\mathrm{A}^{\prime}$ with a set of multipliers (3.3), then $C_{0}$ satisfies condition $\mathrm{A}^{\prime}$ with every set of multipliers$$
i^{0}, \quad \bar{l}^{\sigma}, \quad \bar{m}^{\beta}(a, y)
$$

of class $C^{\prime}$ such that

$$
\bar{l}^{0}=l^{0}, \quad \bar{l}^{\sigma}=l^{\sigma}, \quad \bar{m}^{\beta}(a, y)=m^{\beta}(a, y)
$$

on $C_{0}$. Moreover for every admissible variation $\gamma$ we have $J_{2}(\gamma)=\vec{J}_{2}(\gamma)$, where $J_{2}(\gamma)$ and $\bar{J}_{2}(\gamma)$ are the second variations on $C_{0}$ of the functions $J(C)$ and $\bar{J}(C)$ determined by the multipliers (3.3) and (3.4) respectively.

This result is readily verified with help of Corollary 2 to Theorem 9.3 of the first paper and the fact that $\phi^{\beta}=0$ on $C_{0}$ and $\Phi^{\beta}=0$ for admissible variations.

CoROllary. In the conditions $\mathrm{A}^{\prime}$ and $\mathrm{B}^{\prime}$ for $C_{0}$ only multipliers of the form

$$
l^{0}, \quad l \sigma, \quad m^{\beta}(y)
$$

need be considered. This is also true in condition $\mathrm{B}$ provided $C_{0}$ satisfies condition $\mathrm{A}^{\prime}$ with a set of multipliers (3.5).

Throughout the present paper we shall have occasion to use a special function $K\left(C, C_{0}\right)$ which can be defined as follows: Let the $\operatorname{arcs} C$ and $C_{0}$ be represented in the forms
C:
$a, \quad y(t)$
$(0 \leqq t \leqq 1)$
$C_{0}$ :
$a_{0}, \quad y_{0}(t)$
$(0 \leqq t \leqq 1)$

such that almost everywhere on $0 \leqq t \leqq 1$ one has

(') E. J. McShane, loc. cit. p. 363. 


$$
|\dot{y}(t)|=L(C), \quad\left|\dot{y}_{0}(t)\right|=L\left(C_{0}\right),
$$

where $L(C)$ is the length of $C$. The function $K\left(C, C_{0}\right)$ is defined by the formula

$$
K\left(C, C_{0}\right)=\left|a-a_{0}\right|^{2}+\max _{0 \leqq t \leqq 1}\left|y(t)-y_{0}(t)\right|^{2}+\int_{0}^{1}\left|\dot{y}(t)-\dot{y}_{0}(t)\right|^{2} d t .
$$

Concerning this function we have the following useful result which was established in the second paper $\left.{ }^{5}\right)$.

Lemma 3.3. Suppose that $C_{0}$ satisfies the condition $\mathrm{A}^{\prime}$ with a set of multipliers (3.3) and let $\left\{C_{q}\right\}$ be a sequence of admissible arcs converging to $C_{0}$ in the sense that given a neighborhood $\mathfrak{F}$ of $C_{0}$ in ay-space there is an integer $q_{0}$ such that when $q \geqq q_{0}$, then $C_{q}$ is in $\mathfrak{F}$. If the function $J(C)$ determined by these multipliers is such that

$$
\limsup _{q=\infty} J\left(C_{q}\right) \leqq J\left(C_{0}\right)
$$

then

$$
\lim _{q=\infty} K\left(C_{q}, C_{0}\right)=0 \text {. }
$$

In the proofs of Theorems 4.1 and 4.2 given below it will be convenient to assume that the functions $T^{i 1}(a), T^{i 2}(a)$ appearing in the end conditions (2.2) are linear. This can always be done since our problem is clearly equivalent to that of finding in the class of arcs

$C: \quad a^{h}, \quad b^{i}, \quad c^{i}, \quad y^{i}(t) \quad\left(t^{1} \leqq t \leqq t^{2} ; h=1, \cdots, r ; i=0,1, \cdots, n\right)$ satisfying the equations

$$
\begin{gathered}
\phi^{\beta}(a, y, \dot{y})=0 \quad(\beta=1, \cdots, m<n), \\
y^{i}\left(t^{1}\right)=b^{i}, \quad y^{i}\left(t^{2}\right)=c^{i}, \\
I^{\sigma}(C)=g^{\sigma}(a)+\int_{C} f^{\sigma}(a, y, \dot{y}) d t=0 \quad(\sigma=1, \cdots, s), \\
I^{s+i+1}(C)=T^{i 1}(a)-b^{i}=0, \\
I^{s+n+i+2}(C)=T^{i 2}(a)-c^{i}=0
\end{gathered}
$$

one which minimizes the integral

$$
I(C)=g(a)+\int_{C} f(a, y, \dot{y}) d t .
$$

It is easy to see that conditions $\mathrm{A}^{\prime}$ and $\mathrm{B}^{\prime}$ for $C_{0}$ are equivalent in the two

(5) M. R. Hestenes, The theorem of Lindeberg in the calculus of variations, Trans. Amer. Math. Soc. vol. 60 (1946) pp. 72-92. This paper will be referred to as the "second paper." 
cases as well as the concept of strong and weak relative minima. We shall accordingly assume that end conditions (2.2) are linear whenever it is convenient to do so.

4. The sufficiency theorem. The principal theorem in the present paper can be stated as follows:

THEOREM 4.1. If the arc $C_{0}$ satisfies condition $\mathrm{B}^{\prime}$ as well as those described in $\$ 2$, then there is a neighborhood of $C_{0}$ in ay-space and a constant $\epsilon>0$ such that the inequality

$$
I(C)-I\left(C_{0}\right) \geqq \min \left[\epsilon, \epsilon K\left(C, C_{0}\right)\right] .
$$

holds for every admissible arc $C$ in $\mathfrak{F}$, where $K\left(C, C_{0}\right)$ is defined by (3.6).

This theorem is an extension of one conjectured by McShane $\left(^{6}\right)$ and is an immediate consequence of the following theorem for an arc $C_{0}$ having the properties described in $\$ 2$.

Theorem 4.2. Suppose the arc $C_{0}$ satisfies condition $\mathrm{A}^{\prime}$ with a set of multipliers

$$
l^{0} \geqq 0, \quad l^{\sigma}, \quad m^{\beta}(a, y)
$$

and that the conclusion described in Theorem 4.1 fails to hold. Then there exists an admissible variation $\gamma_{0}$ that is not essentially null such that given any set of multipliers (4.2) with which $C_{0}$ satisfies condition A the second variation $J_{2}\left(\gamma_{0}\right)$ of the function $J(C)$ determined by these multipliers has $J_{2}\left(\gamma_{0}\right) \leqq 0$.

If the conclusion of Theorem 4.2 holds, then $C_{0}$ cannot satisfy condition $\mathrm{B}^{\prime}$. On the other hand if $C_{0}$ satisfies condition $\mathrm{A}^{\prime}$ with a set of multipliers (4.2) and the conclusion of Theorem 4.2 fails to hold, then $C_{0}$ satisfies condition $B$ and hence also condition $B^{\prime}$, by virtue of Lemma 3.1. Thus for an arc $C_{0}$ that satisfies condition $\mathrm{A}^{\prime}$ with a set of multipliers (4.2), Theorems 4.1 and 4.2 are equivalent.

The next seven sections will be devoted to the proof of Theorem 4.2 and hence of Theorem 4.1. As a consequence of Theorem 4.1 we have the following analogue of a theorem of Osgood.

Theorem 4.3. If the arc $C_{0}$ satisfies the condition $\mathrm{B}^{\prime}$ as well as those given in $\$ 2$, there is a neighborhood $\mathfrak{F}$ of $C_{0}$ in ay-space such that given a neighborhood $\mho_{1}$ of $C_{0}$ interior to $\Im_{\text {there }}$ is a constant $\rho>0$ such that the inequality

$$
I(C)-I\left(C_{0}\right)>\rho
$$

holds for every admissible arc $C$ in $\mathfrak{F}_{\text {that }}$ is not in $\mathfrak{F}_{1}$.

For let $\mathfrak{F}$ and $\epsilon$ be chosen as described in Theorem 4.1 and let $\mathfrak{F}_{1}$ be a

(6) Loc. cit. p. 346. 
neighborhood $C_{0}$ interior to $\mathfrak{F}$. From the definition of $K\left(C, C_{0}\right)$ it follows that there is a positive constant $\tau$ such that the relation

$$
K\left(C, C_{0}\right)>\tau
$$

holds for every admissible arc $C$ not in $\mathfrak{\mho}_{1}$. Choose $\rho$ so that $\rho<\epsilon, \rho<\epsilon \tau$. The inequality (4.3) then follows from (4.1).

5. The variation $\gamma_{0}$. In $\$ \S 5-11$ it will be assumed that the hypotheses of Theorem 4.2 hold. It will also be assumed that the functions $T^{i 1}(a)$ and $T^{i 2}(a)$ appearing in the end conditions (2.2) are linear. In view of the remark at the end of $\$ 3$ no generality is lost by this assumption. Since the conclusion of Theorem 4.1 fails to hold there is for every integer $q$ an admissible arc $C_{q}$ in the $(1 / q)$-neighborhood of $C_{0}$ in $a y$-space such that the inequality

$$
I\left(C_{q}\right)-I\left(C_{0}\right)<\min \left[1 / q,(1 / q) K\left(C_{q}, C_{0}\right)\right]
$$

holds. Since $K\left(C_{0}, C_{0}\right)=0$, this inequality could not hold with $C_{q}=C_{0}$. Hence $C_{q} \neq C_{0}$. Throughout the proof of Theorem 4.2 we shall suppose that the arcs $C_{q}(q=0,1,2, \cdots)$ have been given the representation

$C_{q}: \quad a_{q}, \quad y_{q}(t) \quad(0 \leqq t \leqq 1 ; q=0,1, \cdots)$

such that

$$
\left|\dot{y}_{q}(t)\right|=L\left(C_{q}\right) \quad(q=0,1, \cdots)
$$

for almost all values of $t$ on $0 \leqq t \leqq 1$. Here $L\left(C_{q}\right)$ is the length of $C_{q}$. The representation

$C_{0}$ :

$a_{0}, \quad y_{0}(t)$

$(0 \leqq t \leqq 1)$

of $C_{0}$ is of class $C^{\prime \prime}$ on $0 \leqq t \leqq 1$.

Lemma 5.1. The sequence $\left\{C_{q}\right\}$ of admissible arcs described above can be chosen so that

$$
\begin{array}{crl}
\lim _{q=\infty} a_{q}=a_{0}, \quad \lim _{q=\infty} y_{q}(t)=y_{0}(t) \quad \text { uniformly on } & (0 \leqq t \leqq 1), \\
\lim _{q=\infty} \dot{y}_{q}(t)=\dot{y}_{0}(t) & \text { almost uniformly on } & (0 \leqq t \leqq 1), \\
\lim _{q=\infty} k_{q}=0, & 0<k_{q}<1,
\end{array}
$$

where $k_{q}$ is the positive root of the equation

$$
\begin{aligned}
k_{q}^{2}=K\left(C_{q}, C_{0}\right)= & \left|a_{q}-a_{0}\right|^{2}+\max _{0 \leqq t 1}\left|y_{q}(t)-y_{0}(t)\right|^{2} \\
& +\int_{0}^{1}\left|\dot{y}_{q}(t)-\dot{y}_{0}(t)\right|^{2} d t .
\end{aligned}
$$


In order to prove this result let

$$
l^{0}=0 \text { or } 1 ; \quad l^{\sigma}, \quad m^{\beta}(a, y)
$$

be a set of multipliers with which $C_{0}$ satisfies condition $\mathrm{A}^{\prime}$ and let $J(C)$ be the corresponding function (2.12). Then either $J\left(C_{q}\right)=I\left(C_{q}\right)$ or else $J\left(C_{q}\right)$ $=J\left(C_{0}\right)=0$. In either event we have by (5.1)

$$
J\left(C_{q}\right)-J\left(C_{0}\right) \leqq \min \left[1 / q, k_{q}^{2} / q\right],
$$

and hence

$$
\limsup _{q=\infty} J\left(C_{q}\right) \leqq J\left(C_{0}\right) .
$$

By Lemma 3.3 it follows that $\lim _{q-\infty} k_{q}=0$. We can accordingly suppose that $k_{q}<1$. The condition (5.5) therefore holds. From (5.5) and (5.6) it is seen that (5.3) holds. Moreover

$$
\lim _{q=\infty} \int_{0}^{1}\left|\dot{y}_{q}(t)-\dot{y}_{0}(t)\right|^{2} d t=0,
$$

and hence $\lim _{q-\infty} \dot{y}_{q}(t)=\dot{y}_{0}(t)$ in measure on $0 \leqq t \leqq 1$. A subsequence, which we again denote by $\left\{C_{q}\right\}$, accordingly has the property (5.4). This proves the lemma.

In the sequel it will be assumed that the sequence $\left\{C_{q}\right\}$ has been chosen so as to have the properties described in Lemma 5.1. As a further result we have the following lemma.

LEMMa 5.2. For every set of multipliers

$$
l^{0}=0 \text { or } 1, \quad l^{\sigma}, \quad m^{\beta}(a, y),
$$

the corresponding function $J(C)$ defined by (2.12) satisfies the relation

$$
J\left(C_{q}\right)-J\left(C_{0}\right) \leqq k_{q}^{2} / q \quad(q=1,2, \cdots) .
$$

This result follows at once from the relations (5.7), (5.5), and $J(C)=l^{0} I(C)$.

LEMMA 5.3. The variations

$\gamma_{q}: \quad \alpha_{q}=\left(a_{q}-a_{0}\right) / k_{q}, \quad \eta_{q}(t)=\left(y_{q}(t)-y_{0}(t)\right) / k_{q} \quad(0 \leqq t \leqq 1)$ satisfy the condition

$$
\left|\alpha_{q}\right|^{2}+\max _{0 \leqq t \leqq 1}\left|\eta_{q}(t)\right|^{2}+\int_{0}^{1}\left|\dot{\eta}_{q}(t)\right|^{2}=1 .
$$

Consequently, we can select the sequence $\left\{C_{q}\right\}$ so that there exists a variation $\gamma_{0}$ : $\alpha_{0}, \quad \eta_{0}(t)$ $(0 \leqq t \leqq 1)$

satisfying the conditions 


$$
\begin{array}{r}
\lim _{q=\infty} \alpha_{q}=\alpha_{0}, \quad \lim _{q=\infty} \eta_{q}(t)=\eta_{0}(t) \quad \text { uniform } \\
\int_{0}^{1}\left|\dot{\eta}_{0}\right|^{2} d t \leqq \liminf _{q=\infty} \int_{0}^{1}\left|\dot{\eta}_{q}\right|^{2} d t \leqq 1 .
\end{array}
$$

Finally if $g(t)$ is summable together with its square, then

$$
\lim _{Q=\infty} \int_{M} g(t)\left(\eta_{q}^{i}-\eta_{0}^{i}\right) d t=\lim _{q=\infty} \int_{M} g(t)\left(\dot{\eta}_{q}^{i}-\dot{\eta}_{0}^{i}\right) d t=0
$$

for every measurable subset $M$ of $0 \leqq t \leqq 1$.

The relation (5.9) follows from (5.6). It has been shown by McShane( ${ }^{7}$ ) that the condition (5.9) implies the existence of a subsequence of $\left\{\gamma_{q}\right\}$, which we again denote by $\left\{\gamma_{q}\right\}$, and a system $\gamma_{0}$ such that (5.10) holds. Moreover he showed that the functions $\eta_{0}{ }^{i}(t)$ are absolutely continuous and have derivatives that are integrable together with their squares. The system $\gamma_{0}$ accordingly is a variation. We shall show later that $\gamma_{0}$ is an admissible variation. The relations (5.11) and (5.12) were also established by McShane $\left(^{8}\right)$ and Myers( $\left({ }^{9}\right)$.

In what follows it will be assumed that the sequence $\left\{C_{q}\right\}$ has been chosen so as to have the properties described in Lemmas 5.1 and 5.3.

As a further result we have the following lemma.

Lemma 5.4. The variations $\gamma_{q}(q=0,1, \cdots)$ satisfy the variational end conditions (2.6). Moreover for every set of multipliers $l^{0} \geqq 0, l^{\sigma}, m^{\beta}(a, y)$ with which $C_{0}$ satisfies condition A we have

$$
J_{1}\left(\gamma_{q}\right)=0 \quad(q=0,1, \cdots)
$$

where $J_{1}(\gamma)$ is given by (2.13).

6. Three lemmas. The curves $C_{q}$ and $\gamma_{q}$ have associated with them vectors $p_{q}(t)$ and $\pi_{q}(t)$ which we shall now define. Since the matrix $\left\|\phi_{p^{i}}^{\beta}\right\|$ has rank $m$ on $C_{0}$ one can select $\left({ }^{10}\right)$ a set of functions $p^{i}(a, y)$ of class $C^{\prime \prime}$ satisfying the equations

$$
\phi^{\beta}[a, y, p(a, y)]=0 \quad(\beta=1, \cdots, m)
$$

and having the elements $[a, y, p(a, y)]$ on $C_{0}$ when $(a, y)$ is on $C_{0}$. Because the functions $\phi^{\beta}$ are positively homogeneous in $p$ of order one, the functions $p^{i}(a, y)$ can be replaced by functions of the type $k(a, y) p^{i}(a, y)$. We can and shall therefore suppose that these functions have been chosen so that

(7) Loc. cit. pp. 353-355.

(8) Loc. cit. pp. 354-356.

(9) Loc. cit. p. 88.

(10) See Lemma 2.1 of the first paper. 


$$
|p(a, y)|=L\left(C_{0}\right) \text {, }
$$

where $L\left(C_{0}\right)$ is the length of $C_{0}$. By virtue of this choice of $p(a, y)$ and the parametrization of $C_{0}$ we have

$$
\dot{y}_{0}(t)=p\left[a_{0}, y_{0}(t)\right] .
$$

We can now prove the following lemma.

LEMMA 6.1. The vectors $p_{q}(t), \pi_{q}(t)$ defined by the formulas

$$
\begin{aligned}
& p_{q}^{i}(t)=p^{i}\left(a_{q}, y_{q}(t)\right) \\
& \pi_{0}^{i}(t)=p_{a^{k}}^{i}\left(a_{0}, y_{0}(t)\right) \alpha_{0}^{h}+p_{y^{j}}^{i}\left(a_{0}, y_{0}(t)\right) \eta_{0}^{i}(t), \\
& \pi_{q}^{i}(t)=\left(p_{q}^{i}(t)-p_{0}^{i}(t)\right) / k_{q} \\
& (q=0,1, \cdots), \\
& (q=1,2, \cdots)
\end{aligned}
$$

satisfy the relations

$$
\begin{aligned}
& \phi^{\beta}\left(a_{q}, y_{q}(t), p_{q}(t)\right)=0, \quad\left|p_{q}(t)\right|=L\left(C_{0}\right), \\
& 0 \leqq t \leqq 1, \\
& \lim _{q=\infty} p_{q}(t)=p_{0}(t)=\dot{y}_{0}(t) \\
& \lim _{a=\infty} \pi_{q}(t)=\pi_{0}(t) \\
& \text { uniformly on } 0 \leqq t \leqq 1 \text {, } \\
& \text { uniformly on } 0 \leqq t \leqq 1 \text {. }
\end{aligned}
$$

The relations (6.3) follow from (6.1) and (6.2). The relation (6.4) is a consequence of (5.3). An application of Taylor's theorem to the difference

$$
p^{i}\left[a_{q}, y_{q}(t)\right]-p^{i}\left[a_{0}, y_{0}(t)\right]
$$

yields a formula of the form

$$
\pi_{q}^{i}(t)=A_{h q}^{i}(t) \alpha_{q}^{h}+B_{j q}^{i}(t) \eta_{q}^{j}(t)
$$

where

$$
\begin{aligned}
& A_{h q}^{i}=\int_{0}^{1} p_{a^{h}}^{i}\left[a_{0}+\theta\left(a_{q}-a_{0}\right), y_{0}+\theta\left(y_{q}-y_{0}\right)\right] d \theta, \\
& B_{j q}^{i}=\int_{0}^{1} p_{y^{j}}^{i}\left[a_{0}+\theta\left(a_{q}-a_{0}\right), y_{0}+\theta\left(y_{q}-y_{0}\right)\right] d \theta .
\end{aligned}
$$

Using the relations (5.3) and (5.10) it is found that the equation (6.5) holds.

By a similar application of Taylor's theorem and the relations (5.3), (5.10), (6.4), (6.5) one obtains the following lemma.

Lemma 6.2. If $\phi(a, y, p)$ is a function of class $C^{\prime}$, then

$$
\lim _{q=\infty} \frac{\phi\left[a_{q}, y_{q}(t), p_{q}(t)\right]-\phi\left[a_{0}, y_{0}(t), p_{0}(t)\right]}{k_{q}}=\Phi\left[t, \alpha_{0}, \eta_{0}(t), \pi_{0}(t)\right]
$$

uniformly on $0 \leqq t \leqq 1$, where 


$$
\Phi(t, \alpha, \eta, \pi)=\phi_{a_{h}} \alpha^{h}+\phi_{y} i \eta^{i}+\phi_{p} i \pi^{i},
$$

the arguments in the derivatives of $\phi$ being $\left[a_{0}, y_{0}(t), \dot{y}_{0}(t)\right]$.

Applying this result to the functions $\phi^{\beta}(a, y, p)$ we have by virtue of (6.3) the following corollary.

COROLLARY. The variation $\gamma_{0}$ satisfies with $\pi_{0}(t)$ the equations

$$
\Phi^{\beta}\left[t, \alpha_{0}, \eta_{0}(t), \pi_{0}(t)\right]=0,
$$

where $\Phi^{\beta}$ is the variation (6.6) of $\phi^{\beta}$.

As a further result we have another lemma.

LEMMA 6.3. If $B_{i q}(t)(q=0,1, \cdots)$ are continuous functions such that

$$
\lim _{q=\infty} B_{i q}(t)=B_{i 0}(t)
$$
uniformly on $0 \leqq t \leqq 1$,

then for every measurable subset $M$ of $0 \leqq t \leqq 1$ one has

$$
\lim _{q=\infty} \int_{M} B_{i q}\left(\dot{\eta}_{q}^{i}-\pi_{q}^{i}\right) d t=\int_{M} B_{i 0}\left(\dot{\eta}_{0}^{i}-\pi_{0}^{i}\right) d t .
$$

In order to prove this result observe first that by virtue of (6.8) and (6.5) we have

$$
\lim _{q=\infty} \int_{M} B_{i q} \pi_{q}^{i} d t=\int_{M} B_{i 0} \pi_{0}^{i} d t
$$

It remains therefore to show that

$$
\lim _{q=\infty} \int_{M} B_{i q} \dot{\eta}_{q}^{i} d t=\int_{M} B_{i 0} \dot{\dot{\eta}_{0}} d t
$$

To this end observe that

$$
\int_{M} \dot{B}_{i q} \dot{\eta}_{q}^{i} d t=\int_{M} B_{i 0} \dot{\eta}_{q}^{i} d t+\int_{M}\left(B_{i q}-B_{i 0}\right) \dot{\eta}_{q}^{i} d t .
$$

By the inequality of Schwarz and (5.9) we have

$$
\begin{aligned}
\left|\int_{M}\left(B_{i q}-B_{i 0}\right) \dot{\eta}_{q}^{i} d t\right|^{2} & \leqq\left[\int_{M}\left|B_{q}-B_{0}\right|^{2} d t\right]\left[\int_{M}\left|\dot{\eta}_{q}\right|^{2} d t\right] \\
& \leqq \int_{0}^{1}\left|B_{q}-B_{0}\right|^{2} d t .
\end{aligned}
$$

It follows from (6.8) that the last term in (6.11) converges to zero. By virtue of (5.12) we have 


$$
\lim _{Q=\infty} \int_{M} B_{i 0} \dot{\eta}_{Q}^{i} d t=\int_{M} B_{i 0} \dot{\eta}_{0}^{i} d t
$$

It follows from (6.11) that (6.10) holds and Lemma 6.3 is established.

7. Properties of $H(C, M)$. Consider an admissible arc $C$ parameterized on $0 \leqq t \leqq 1$ so that $|\dot{y}|=L(C)$. Given a measurable subset $M$ of $0 \leqq t \leqq 1$, a function $\theta(a)$ of class $C^{\prime}$ and an admissible function $H(a, y, p)$ of class $C^{\prime}$ we define $H(C, M)$ by the formula

$$
H(C, M)=\theta(a)+\int_{M} H(a, y(t), \dot{y}(t)) d t .
$$

Similarly using the functions $p^{i}(a, y)$ chosen in the last section we set

$$
\begin{aligned}
& H^{*}(C, M)=\theta(a)+\int_{M} H_{p^{i}}[a, y(t), p(a, y(t))] \dot{y}_{i}(t) d t, \\
& E_{H}^{*}(C, M)=\int_{M} E_{H}[a, y(t), p(a, y(t)), \dot{y}(t)] d t .
\end{aligned}
$$

We have accordingly

$$
H(C, M)=H^{*}(C, M)+E_{H}^{*}(C, M) .
$$

Finally we define $H_{1}(\gamma, M)$ by the equation

$$
H_{1}(\gamma, M)=\theta_{a^{h} \alpha^{h}}+\int_{M}\left\{H_{a^{h} \alpha^{h}}+H_{y^{i} \eta^{i}}+H_{p^{i} \eta^{i}}\right\} d t,
$$

where the derivatives of $\theta(a)$ and $H(a, y, p)$ are evaluated along $C_{0}$. When $M$ is the interval $0 \leqq t \leqq 1$, the values $H(C, M), H^{*}(C, M), E_{H}^{*}(C, M)$, $H_{1}(\gamma, M)$ will be denoted respectively by $H(C), H^{*}(C), E_{H}{ }^{*}(C), H_{1}(\gamma)$. These notations are consistent with the ones used earlier and equation (7.1) takes the form $H(C)=H^{*}(C)+E_{H}^{*}(C)$.

As a first result we have the following lemma.

\section{LEMma 7.1. The relation}

$$
\lim _{q=\infty} \frac{H^{*}\left(C_{q}, M\right)-H^{*}\left(C_{0}, M\right)}{k_{q}}=H_{1}\left(\gamma_{0}, M\right)
$$

holds for every measurable subset $M$ of $0 \leqq t \leqq 1$.

In order to prove this result observe that the integrand of $H^{*}\left(C_{g}, M\right)$ can be written in the form

$$
H\left(a_{q}, y_{q}, p_{q}\right)+\left(\dot{y}_{q}^{i}-p_{q}^{i}\right) H_{p^{i}}\left(a_{q}, y_{q}, p_{q}\right) .
$$

By virtue of Lemma 6.2 we have 


$$
\begin{gathered}
\lim _{q=\infty} \frac{\theta\left(a_{q}\right)-\theta\left(a_{0}\right)}{k_{q}}=\theta_{a_{h}}\left(a_{0}\right) \alpha_{0}^{h}, \\
\lim _{q=\infty} \frac{H\left(a_{q}, y_{q}, p_{q}\right)-H\left(a_{0}, y_{0}, p_{0}\right)}{k_{q}}=H_{a^{h} \alpha}{ }^{h}+H_{y^{i} \eta_{0}^{i}}^{i}+H_{p^{i} \pi_{0}^{i}}^{i}
\end{gathered}
$$

uniformly on $M$. Here the arguments in the derivatives of $H$ are $\left[a_{0}, y_{0}(t)\right.$, $\left.\dot{y}_{0}(t)\right]$. Since $\dot{y}_{0}{ }^{i}(t)=p_{0}{ }^{i}(t)$ it follows from the definitions of $\eta_{q}{ }^{i}$ and $\pi_{q}{ }^{i}$ that

$$
\left(\dot{y}_{q}^{i}-p_{q}^{i}\right) / k_{q}=\left(\dot{\eta}_{q}^{i}-\pi_{q}^{i}\right) \quad(q=1,2, \cdots) .
$$

An application of Lemma 6.3 with $B_{i q}=H_{p^{i}}\left(a_{q}, y_{q}, p_{q}\right)$ yields

$$
\lim _{q=\infty} \frac{1}{k_{q}} \int_{M}\left(\dot{y}_{q}^{i}-p_{q}^{i}\right) H_{p^{i}}\left(a_{q}, y_{q}, p_{q}\right) d t=\int_{M}\left(\dot{\eta}_{0}^{i}-\pi_{0}^{i}\right) H_{p^{i}}\left(a_{0}, y_{0}, p_{0}\right) d t .
$$

If we combine this result with (7.3) it is readily seen that (7.2) holds. This proves Lemma 7.1.

LEMma 7.2. For every set of multipliers

$$
l^{0}=0 \text { or } 1, \quad l^{\sigma}, \quad m^{\beta}(a, y)
$$

with which the arc $C_{0}$ satisfies condition $A$, the corresponding functions $J(C)$, $J^{*}(C), E_{F}^{*}(C)$ satisfy the relations

$$
\lim _{q=\infty} \frac{J\left(C_{q}\right)-J\left(C_{0}\right)}{k_{q}}=\lim _{q=\infty} \frac{J^{*}\left(C_{q}\right)-J^{*}\left(C_{0}\right)}{k_{q}}=\lim _{q=\infty} \frac{1}{k_{q}} E_{F}^{*}\left(C_{q}\right)=0 .
$$

In order to prove this result recall that

$$
J\left(C_{q}\right)=J^{*}\left(C_{q}\right)+E_{F}^{*}\left(C_{q}\right) .
$$

Since $\dot{y}_{0}(t)=p_{0}(t)$ we have $E_{F}^{*}\left(C_{0}\right)=0$ and hence $J\left(C_{0}\right)=J^{*}\left(C_{0}\right)$. Consequently

$$
J\left(C_{q}\right)-J\left(C_{0}\right)=J^{*}\left(C_{q}\right)-J^{*}\left(C_{0}\right)+E_{F}^{*}\left(C_{q}\right) .
$$

Moreover by Lemma 7.1 and the relation $J_{1}\left(\gamma_{0}\right)=0$ given in Lemma 5.4 we have

$$
\lim _{q=\infty} \frac{J^{*}\left(C_{q}\right)-J^{*}\left(C_{0}\right)}{k_{q}}=J_{1}\left(\gamma_{0}\right)=0 .
$$

By virtue of (5.8) we have accordingly

$$
\underset{q=\infty}{\limsup } \frac{J\left(C_{q}\right)-J\left(C_{0}\right)}{k_{q}}=\limsup _{q=\infty} \frac{1}{k_{q}} E_{F}^{*}\left(C_{q}\right) \leqq 0 .
$$

From this result it follows that the lemma will be established if we show that 


$$
\liminf _{q=\infty} \frac{1}{k_{q}} E_{F}^{*}\left(C_{q}\right) \geqq 0 .
$$

Since $k_{q}>0$ it is sufficient to show that there is an integer $q_{0}$ such that when $g>q_{0}$ we have

$$
E_{F}^{*}\left(C_{q}\right)=\int_{0}^{1} E_{F}\left[a_{q}, y_{q}(t), p_{q}(t), \dot{y}_{q}(t)\right] d t \geqq 0 \quad\left(q \geqq q_{0}\right) .
$$

In order to establish this inequality recall that by virtue of our hypothesis there exists a neighborhood $\mathfrak{D}_{0}$ of $C_{0}$ relative to the class $\mathfrak{D}$ of differentially admissible elements $(a, y, p)$ such that the inequality

$$
E_{F}(a, y, p, q) \geqq 0
$$

holds whenever $(a, y, p)$ is in $\mathfrak{D}_{0}$ and $(a, y, q)$ is in $\mathfrak{D}$. By virtue of the relations (5.3), (6.3) and (6.4) we can select $q_{0}$ such that when $q \geqq q_{0}$ we have $\left(a_{q}, y_{q}(t)\right.$, $\left.p_{q}(t)\right)$ in $\mathfrak{D}_{0}$. Moreover since $C_{q}$ is admissible the elements $\left(a_{q}, y_{q}(t), \dot{y}_{q}(t)\right)$ are in $\mathfrak{D}$ for almost all values of $t$ on $0 \leqq t \leqq 1$. It follows that when $q \geqq q_{0}$ the integrand appearing in (7.7) is non-negative for almost all values of $t$ on $0 \leqq t \leqq 1$. The inequality (7.7) accordingly holds and Lemma 7.2 is established.

Lemma 7.3. Let $F(a, y, p)=l^{0} f+l^{\sigma} f^{\sigma}+m^{\beta} \phi^{\beta}$ be the admissible function determined by $a$ set of multipliers (7.5) with which $C_{0}$ satisfies condition $\mathrm{A}$. If $H(a, y, p)$ is $E$-dominated by $F(a, y, p)$ near $C_{0}$ on $\mathfrak{D}$, then

$$
\lim _{q=\infty} \frac{H\left(C_{q}, M\right)-H\left(C_{0}, M\right)}{k_{q}}=H_{1}\left(\gamma_{0}, M\right)
$$

for every measurable subset $\dot{M}$ of $0 \leqq t \leqq 1$.

In order to prove this result observe that since $H(a, y, p)$ is $E$-dominated by $F$ near $C_{0}$ on $\mathfrak{D}$, there is a constant $b>0$ and an integer $q_{0}$ such that when $q>q_{0}$ the inequality

$$
\left|E_{H}\left[a_{q}, y_{q}(t), p_{q}(t), \dot{y}_{q}(t)\right]\right| \leqq b E_{F}\left[a_{q}, y_{q}(t), p_{q}(t), \dot{y}_{q}(t)\right]
$$

holds for almost all values of $t$ on $0 \leqq t \leqq 1$. This result follows from (5.3), (6.3), (6.4) and the definition of $E$-dominance. We have accordingly

$$
\begin{aligned}
\left|E_{H}^{*}\left(C_{q}, M\right)\right| & \leqq \int_{M}\left|E_{H}\left(a_{q}, y_{q}, p_{q}, \dot{y}_{q}\right)\right| d t \\
& \leqq \int_{0}^{1}\left|E_{H}\left(a_{q}, y_{q}, p_{q}, \dot{y}_{q}\right)\right| d t \\
& \leqq b \int_{0}^{1} E_{F}\left(a_{q}, y_{q}, p_{q}, \dot{y}_{q}\right) d t=b E_{F}^{*}\left(C_{q}\right) .
\end{aligned}
$$


Combining this result with (7.6) it is seen that

$$
\lim _{q=\infty} \frac{1}{k_{q}} E_{H}^{*}\left(C_{q}, M\right)=0 .
$$

Using (7.1) together with the relation $E_{H}^{*}\left(C_{0}, M\right)=0$ we have the further formula

(7.10) $\quad H\left(C_{q}, M\right)-H\left(C_{0}, M\right)=H^{*}\left(C_{q}, M\right)-H^{*}\left(C_{0}, M\right)+E_{H}^{*}\left(C_{q}, M\right)$.

The relation (7.8) follows readily from (7.10), (7.2) and (7.9). This proves Lemma 7.3.

8. Admissibility of $\gamma_{0}$. The purpose of the present section is to establish the following lemma.

LEMMA 8.1. The variation $\gamma_{0}$ described in $\$ 5$ is admissible and satisfies the equation

$$
\dot{y}_{0}^{i}(t) \dot{\eta}_{0}^{i}(t)=\text { constant }
$$

for almost all values of $t$ on $0 \leqq t \leqq 1$.

In order to prove this result select $F(a, y, p)$ as described in Condition $\mathrm{A}^{\prime}$ for $C_{0}$. Then $L(p)=|p|, f^{\sigma}(a, y, p)$ are $E$-dominated by $F(a, y, p)$ near $C_{0}$ on $\mathfrak{D}$. In fact by Theorem 4.3 of the first paper the functions $\phi^{\beta}(a, y, p)$ are also $E$-dominated by $F(a, y, p)$ near $C_{0}$ on $\mathfrak{D}$. Consequently if we select

$$
H(C, M)=\int_{M} \phi^{\beta}(a, y, \dot{y}) d t
$$

then $H\left(C_{q}, M\right)=H\left(C_{0}, M\right)=0$ and by Lemma 7.3

$$
\int_{M} \Phi^{\beta}\left(t, \alpha_{0}, \eta, \dot{\eta}\right) d t=0
$$

for every measurable subset $M$ of $0 \leqq t \leqq 1$. Hence

$$
\Phi^{\beta}\left(t, \alpha_{0}, \eta_{0}(t), \dot{\eta}_{0}(t)\right)=0
$$

for almost all values of $t$ on $0 \leqq t \leqq 1$, that is, the variation $\gamma_{0}$ is differentially admissible.

In order to show that $\gamma_{0}$ is also admissible let $M$ be the interval $0 \leqq t \leqq 1$ and choose $H(C, M)=I^{\sigma}(C)$. We then have $H\left(C_{q}, M\right)=I^{\sigma}\left(C_{q}\right)=0 \quad(q=0$, $1, \cdots)$ since $C_{q}$ is admissible. Moreover $H_{1}(\gamma, M)$ is identical with the first variation $I_{1}{ }^{\sigma}(\gamma)$ of $I^{\sigma}(C)$ on $C_{0}$. It follows from (7.8) that $I_{1}{ }^{\sigma}\left(\gamma_{0}\right)=0$. Furthermore by Lemma 5.4 the variation $\gamma_{0}$ satisfies the variational end conditions (2.6). The variation $\gamma_{0}$ is therefore admissible, as was to be proved.

It remains to show that the relation (8.1) holds To this end we select 


$$
H\left(C_{q}, M\right)=\int_{0}^{t} L\left(\dot{y}_{q}\right) d t=\int_{0}^{t}\left|\dot{y}_{q}\right| d t=L\left(C_{q}\right) t
$$

the last equality holding by virtue of our parametrization of $C_{q}$. We then have

$$
H_{1}\left(\gamma_{0}, M\right)=\int_{0}^{t} L_{p^{i}}\left(\dot{y}_{0}\right) \dot{\eta_{0}} d t=\frac{1}{L\left(C_{0}\right)} \int_{0}^{t} \dot{y}_{0}^{i} \dot{\eta}_{0}^{i} d t
$$

Moreover by Lemma 7.3

$$
H_{1}\left(\gamma_{0}, M\right)=t \lim _{q=\infty} \frac{L\left(C_{q}\right)-L\left(C_{0}\right)}{k_{q}} .
$$

Consequently

$$
\int_{0}^{t} \dot{y}_{0}^{i} \dot{\eta}_{0} d t=t L\left(C_{0}\right) \lim _{q=\infty} \frac{L\left(C_{q}\right)-L\left(C_{0}\right)}{k_{q}} .
$$

The equation (8.1) follows from (8.2) by differentiation. This proves Lemma 8.1.

Setting $t=1$ in (8.2) one obtains the formula

$$
\lim _{q=\infty} \frac{L\left(C_{q}\right)-L\left(C_{0}\right)}{k_{q}}=\frac{1}{L\left(C_{0}\right)} \int_{0}^{1} \dot{y}_{0}^{i} \dot{\eta}_{0}^{i} d t,
$$

a result that will be found useful in $\$ 11$.

9. Second order terms. In the following lemma we are concerned with the second variation $J_{2}^{*}(\gamma)$ of the functions

$$
J^{*}(C)=G(a, l)+\int_{C} F_{p^{i}}(a, y, p(a, y), l, m(a, y)) d y^{i},
$$

where $F$ and $G$ are the functions (2.11) determined by a set of multipliers $l^{0}=0$ or $1, l^{\sigma}, m^{\beta}(a, y)$ of class $C^{\prime}$ with which $C_{0}$ satisfies the Euler equations (2.14) and the transversality condition (2.15). This second variation $J_{2}^{*}(\gamma)$ is given by the formula

$$
J_{2}^{*}(\gamma)=b_{k k} \alpha^{h} \alpha^{k}+\int_{0}^{1}\left\{2 \Omega(t, \alpha, \eta, \pi)+2\left(\dot{\eta}^{i}-\pi^{i}\right) \Omega_{x^{i}}(t, \alpha, \eta, \pi)\right\} d t
$$

where $\pi^{i}$ denotes the variation of $p^{i}(a, y)$ along $C_{0}$. We shall be interested only in the case when $\gamma=\gamma_{0}$. In this event $\pi^{i}=\pi_{0}{ }^{i}(t)$.

LEMMA 9.1. Under the hypotheses described above we have

$$
\lim _{q=\infty} \frac{J^{*}\left(C_{q}\right)-J^{*}\left(C_{0}\right)}{k_{q}^{2}}=2^{-1} J_{2}^{*}\left(\gamma_{0}\right) .
$$


In order to prove this result it will be convenient to denote $F(a, y, p, l, m)$ by $F(a, y, p, m)$. We introduce the following notations

$$
\begin{array}{rlrl}
m_{q}^{\beta}(t) & =m^{\beta}\left(a_{q}, y_{q}(t)\right) & (q=0,1,2, \cdots), \\
\mu_{0}^{\beta}(t) & =m_{a^{k}}^{\beta}\left(a_{0}, y_{0}(t)\right) \alpha_{0}^{h}+m_{y^{i}}^{\beta}\left(a_{0}, y_{0}(t)\right) \eta_{0}^{i}(t), & \\
\mu_{q}^{\beta}(t) & =\left(m_{q}^{\beta}(t)-m_{0}^{\beta}(t)\right) / k_{q} & & (q=1,2, \cdots) .
\end{array}
$$

By the use of (5.3), (5.10) and Taylor's Theorem we have

$$
\lim _{q=\infty} m_{q}^{\beta}(t)=m_{0}^{\beta}(t), \quad \lim _{q=\infty} \mu_{q}^{\beta}(t)=\mu_{0}^{\beta}(t)
$$

uniformly on $0 \leqq t \leqq 1$.

Because of the relation $F=p^{i} F_{p^{i}}$ the integrand of $J^{*}\left(C_{q}\right)$ can be written in the form

$$
F\left(a_{q}, y_{q}, p_{q}, m_{q}\right)+\left(\dot{y}_{q}^{i}-p_{q}^{i}\right) F_{p_{i}}\left(a_{q}, y_{q}, p_{q}, m_{q}\right) .
$$

Since $\phi^{\beta}=0$ along $C_{q}$ we have $F\left(a_{q}, y_{q}, p_{q}, m_{q}\right)=F\left(a_{q}, y_{q}, p_{q}, m_{0}\right)$ and hence by Taylor's Theorem applied to the latter function

$$
F\left(a_{q}, y_{q}, p_{q}, m_{q}\right)=F\left(a_{0}, y_{0}, p_{0}, m_{0}\right)+k_{q} F_{1 q}+2^{-1} k_{q}^{2} F_{2 q}
$$

where

$$
F_{1 q}=F_{a^{h} \alpha_{q}^{h}}^{h}+F_{y^{i} \eta_{q}^{i}}^{i}+F_{p^{i} \pi_{q}^{i}}^{i}
$$

and

$$
\lim _{q=\infty} F_{2 q}(t)=2 \Omega\left(t, \alpha_{0}, \eta_{0}(t), \pi_{0}(t)\right)
$$

uniformly on $0 \leqq t \leqq 1$. Similarly

$$
F_{p^{i}}\left(a_{q}, y_{q}, p_{q}, m_{q}\right)=F_{p^{i}}\left(a_{0}, y_{0}, p_{0}, m_{0}\right)+k_{q} B_{i q}
$$

where

$$
\lim _{\boldsymbol{q}=\infty} B_{i q}(t)=\Omega_{\pi^{i}}\left(t, \alpha_{0}, \eta_{0}(t) ; \pi_{0}(t)\right)
$$

uniformly on $0 \leqq t \leqq 1$. Using the relations (9.6), (9.7), (9.9) and

$$
\dot{y}_{0}^{i}=p_{0}^{i}, \quad \dot{y}_{q}^{i}-p_{q}^{i}=k_{q}\left(\dot{\eta}_{q}^{i}-\pi_{q}^{i}\right)
$$

it is seen that the integrand (9.5) of $J^{*}\left(C_{q}\right)$ takes the form

$$
\begin{aligned}
F\left(a_{0}, y_{0}, p_{0}, m_{0}\right)+k_{q}\left\{F_{a^{h} \alpha_{q}}^{h}+F_{y^{i} \eta_{q}^{i}}\right. & \left.+F_{p^{i} \dot{\eta}_{q}}^{i}\right\} \\
& +2^{-1} k_{q}^{2}\left\{F_{2 q}+2\left(\dot{\eta}_{q}^{i}-\pi_{q}^{i}\right) B_{i q}\right\} .
\end{aligned}
$$

Finally, setting $G(a)=G(a, l)$, we have

$$
G\left(a_{q}\right)=G\left(a_{0}\right)+k_{q} G_{a^{h}}\left(a_{0}\right) \alpha_{q}^{h}+2^{-1} k_{q}^{2} G_{2 q}
$$


where

$$
\lim _{q=\infty} G_{2 q}=G_{a^{k} a^{k} \alpha_{0} \alpha_{0}^{k}}^{h k}=b_{h k} \stackrel{h \alpha_{0} \alpha_{0}^{k},}{k}
$$

the last equality holding because of the linearity of the functions $T^{\mathbf{i}}(a)$ and $T^{i 2}(a)$. Because of the relation (9.19) and the formula (9.11) for the integrand of $J^{*}\left(C_{q}\right)$ we have

$$
J^{*}\left(C_{q}\right)-J^{*}\left(C_{0}\right)=k_{q} J_{1}\left(\gamma_{q}\right)+2^{-1} k_{q}^{2} J_{2 q}^{*}
$$

where $J_{1}(\gamma)$ is the first variation (2.13) of $J(C)$ and

$$
J_{2 q}^{*}=G_{2 q}+\int_{0}^{1}\left\{F_{2 q}+2\left(\dot{\eta}_{q}^{i}-\pi_{q}^{i}\right) B_{i q}\right\} d t .
$$

In view of Lemma 5.4 we have $J_{1}\left(\gamma_{q}\right)=0$. Moreover by virtue of $(9.8),(9.10)$, and (9.13) we have

$$
\lim _{q=\infty} J_{2 q}^{*}=J_{2}^{*}\left(\gamma_{0}\right)
$$

Combining this relation with (9.14) and $J_{1}\left(\gamma_{q}\right)=0$ we see that (9.2) holds, as was to be proved.

As an interesting consequence of the result just proved we have the following lemma.

LEMMA 9.2. If $H(a, y, p)$ is an admissible function of the form

$$
H=\theta(a, y, p) \phi^{\beta} \phi^{\beta}
$$

where $\theta$ is of class $C^{\prime \prime}$, then

$$
\lim _{q=\infty} k_{q}^{-2} \int_{0}^{1} E_{H}\left(a_{q}, y_{q}, p_{q}, \dot{y}_{q}\right) d t=0 .
$$

In order to prove this result observe that the only properties of $J(C)$ used in the proof of the last section were that its first variation $J_{1}(\gamma)$ on $C_{0}$ vanished for every admissible variation $\gamma$. The function $J(C)=\int_{c} H d t$ also has this property. Hence Lemma 9.1 is applicable in this case also. Moreover, since $\phi^{\beta}=0$ on $C_{0}$ we have in this event

$$
2 \Omega(t, \alpha, \eta, \pi)=\theta \Phi^{\beta}(t, \alpha, \eta, \pi) \Phi^{\beta}(t, \alpha, \eta, \pi) .
$$

Hence by (6.7) and Lemma 9.1 we have

$$
\lim _{q=\infty} \frac{J^{*}\left(C_{q}\right)-J^{*}\left(C_{0}\right)}{k_{q}^{2}}=2^{-1} J_{2}^{*}\left(\gamma_{0}\right)=0 .
$$

The lemma follows from this relation, the equations $J\left(C_{q}\right) \doteq J\left(C_{0}\right)=0$, and the familiar formula $J\left(C_{q}\right)-J\left(C_{0}\right)=J^{*}\left(C_{q}\right)-J^{*}\left(C_{0}\right)+E_{F}^{*}\left(C_{q}\right)$. 
LEMma 9.3. There exists a constant $N>0$ such that

$$
0 \leqq \liminf _{q=\infty} k_{q}^{-2} E_{L}^{*}\left(C_{q}\right) \leqq \limsup _{q=\infty} k_{q}^{-2} E_{L}^{*}\left(C_{q}\right)<N .
$$

For suppose the multipliers $l^{0}=0, l^{\sigma}, m^{\beta}(a, y)$ have been chosen as described in Condition $A$ for $C_{0}$. Then by the use of the inequality (5.8), the equation

$$
J\left(C_{q}\right)-J\left(C_{0}\right)=J^{*}\left(C_{q}\right)-J^{*}\left(C_{0}\right)+E_{F}^{*}\left(C_{q}\right),
$$

and the limit (9.2), it is seen that

$$
2^{-1} J_{2}^{*}\left(\gamma_{0}\right)+\limsup _{q=\infty} k_{q}^{-2} E_{F}\left(C_{q}\right) \leqq 0 .
$$

By hypothesis $L$ is $E$-dominated by $F$ near $C_{0}$ on $\mathfrak{D}$. Hence there exists a constant $b>0$ such that

$$
E_{L}^{*}\left(C_{q}\right) \leqq b E_{F}^{*}\left(C_{q}\right)
$$

for large values of $q$. Consequently by (9.16) we have

$$
\limsup _{q=\infty} \overrightarrow{k q}_{q}^{-2} E_{L}^{*}\left(C_{q}\right) \leqq-\frac{b}{2} J_{2}^{*}\left(\gamma_{0}\right) \text {. }
$$

Since $E_{L}^{*}\left(C_{q}\right) \geqq 0$ this proves the lemma.

10. A property of $E_{F}{ }^{*}(C)$. The proof of Theorem 3.1 will be completed in the next section. In the proof we shall make use of the following lemma.

LEMMA 10.1. If $F(a, y, p)$ is an admissible function that $E$-dominates $H=0$ near $C_{0}$ on $\mathfrak{D}$, then

$$
\liminf _{q=\infty} k_{q}^{-2} E_{F}^{*}\left(C_{q}\right) \geqq 2^{-1} \int_{0}^{1} F_{p^{i} p^{j}}\left(\dot{\eta}_{0}^{i}-\pi_{0}^{i}\right)\left(\dot{\eta}_{0}^{j}-\pi_{0}^{j}\right) d t \geqq 0
$$

where the arguments of the derivatives of $F$ on the right are the values $\left[a_{0}, y_{0}(t)\right.$, $\left.\dot{y}_{0}(t)\right]$ on $C_{0}$.

In order to prove this result let $M$ be a measurable subset on which the sequence $\dot{y}_{q}^{i}(t)$ converges uniformly to $\dot{y}_{0}{ }^{i}(t)$. For $q$ sufficiently large we have by Taylor's Theorem

$$
E_{F}\left(a_{q}, y_{q}, p_{q}, \dot{y}_{q}\right)=A_{i j_{q}}(t)\left(\dot{y}_{q}^{i}-p_{q}^{i}\right)\left(\dot{y}_{q}^{j}-p_{q}^{j}\right)
$$

where

$$
A_{i j q}(t)=\int_{0}^{1}(1-s) F_{p^{i} p^{i}}\left[a_{q}, y_{q}, p_{q}+s\left(\dot{y}_{q}-p_{q}\right)\right] d s
$$

If we set 


$$
A_{i j}(t)=2^{-1} F_{p^{i} p^{i}}\left[a_{0}, y_{0}(t), \dot{y}_{0}(t)\right]
$$

then

$$
\lim _{a=\infty} A_{i j q}(t)=A_{i j}(t)
$$

uniformly on $M$. For convenience set

$$
\xi_{q}^{i}=\dot{\eta}_{q}^{i}-\pi_{q}^{i} \quad(q=0,1,2, \cdots) .
$$

By the use of (5.9) and (6.5) it is seen that

$$
\int_{M}\left|\xi_{q}\right|^{2} d t \leqq \int_{0}^{1}\left|\xi_{q}\right|^{2} d t \leqq N \quad(q=1,2, \cdots)
$$

where $N$ is a suitably chosen constant. It should be observed that by virtue of equations (6.7) and the admissibility of $\gamma_{0}$ we have

$$
\phi_{p^{i}}^{\beta}\left(a_{0}, y_{0}, p_{0}\right) \xi_{0}^{i}=0
$$

almost everywhere on $0 \leqq t \leqq 1$.

Consider now the equation

$$
\int_{M} A_{i j q} \xi_{q}^{i} \xi_{q}^{j} d t=\int_{M} A_{i j} \xi_{q}^{i} \xi_{q}^{j} d t+\int_{M}\left(A_{i j q}-A_{i j}\right) \xi_{q}^{i} \xi_{q}^{j} d t
$$

By virtue of the relations (10.4) and (10.6) the last integral has the limit zero as $q$ becomes infinite. It follows that

$$
\liminf _{Q=\infty} \int_{M} A_{i j_{q}} \xi_{q}^{i} \xi_{q}^{j} d t=\liminf _{e=\infty} \int_{M} A_{i j} \xi_{q}^{i} \xi_{q}^{j} d t
$$

Moreover by the use of (5.12) and the definition (10.5) of $\xi_{q}{ }^{i}$ it is seen that

$$
\lim _{q=\infty} \int_{M} A_{i j} \xi_{0}^{i} \xi_{q}^{j} d t=\int_{M} A_{i j} \xi_{0}^{i} \xi_{0}^{j} d t
$$

We have accordingly

$$
\begin{aligned}
\underset{q=\infty}{\liminf } \int_{M} A_{i j} \xi_{q}^{i} \xi_{q}^{j} d t= & \int_{M} A_{i j} \xi_{0}^{i} \xi_{0}^{j} d t \\
& +\liminf _{q=\infty} \int_{M} A_{i j}\left(\xi_{q}^{i}-\xi_{0}^{i}\right)\left(\xi_{q}^{j}-\xi_{0}^{j}\right) d t .
\end{aligned}
$$

We are now in position to prove Lemma 10.1 for the case when (10.10)

$$
F_{p^{i} p^{j} \xi^{i} \xi^{i}} \geqq 0
$$

along $C_{0}$ for every set $\xi$. Then the last term in (10.9) is non-negative. By the 
use of equations (10.2), (10.8), (10.9), and (10.4), it is seen that

$$
\liminf _{q=\infty} k_{q}^{-2} \int_{M} E_{F}\left(a_{q}, y_{q}, p_{q}, \dot{y}_{q}\right) d t \geqq 2^{-1} \int_{M} F_{p^{i} p^{i} \xi_{0}^{i} \xi_{0}^{i} d t .}
$$

Since by hypothesis $E_{F}\left(a_{q}, y_{q}, p_{q}, \dot{y}_{q}\right) \geqq 0$ almost everywhere on $0 \leqq t \leqq 1$ for $q$ sufficiently large, we have

$$
\liminf _{q=\infty} k_{q}^{-2} E_{F}^{*}\left(C_{q}\right) \geqq 2^{-1} \int_{M} F_{p^{i} p^{i} \xi_{0}^{i} \xi_{0}^{j} d t}
$$

Inasmuch as the sequence $\dot{y}_{q}{ }^{i}(t)$ converges to $\dot{y}_{0}{ }^{i}(t)$ almost uniformly on $0 \leqq t \leqq 1$, it follows from our choice of $M$ that this inequality also holds when $M$ is the interval $0 \leqq t \leqq 1$. This proves the lemma for the case when (10.10) holds.

Consider now the case when the inequality (10.10) holds on $C_{0}$ subject to the conditions $\phi_{p^{\prime}}^{\beta} \xi^{i}=0$, the equality holding only when $\xi=\rho p$. Then by Theorem 3.1 in the first paper there is a function

$$
F^{*}=F+\theta \phi^{\beta} \phi^{\beta}
$$

such that the inequality $(10.10)$ holds with $F$ replaced by $F^{*}$. Consequently

$$
\lim _{q=\infty} \inf _{k_{q}}^{-2} E_{F^{*}}^{*}\left(C_{q}\right) \geqq 2^{-1} \int_{0}^{1} F_{p^{i} p^{i}}^{*} \xi_{Q}^{i} \xi_{0}^{j} d t \geqq 0 .
$$

But $E_{F^{*}}^{*}(C)=E_{F}^{*}(C)+E_{H}^{*}(C)$, where $H=\theta \phi^{\beta} \phi^{\beta}$. Hence by Lemma 9.2 the first member of the relation (10.11) is equal to the first member of (10.1). From (10.7) and the definition of $F^{*}$ it follows that the second members are also equal. Hence Lemma 10.1 holds in this case also.

To prove the lemma as stated observe that our hypotheses imply that (10.10) holds on $C_{0}$ for all solutions $\xi$ of the equations $\phi_{p^{i}}^{\beta} \xi^{i}=0$ on $C_{0}$. The admissible function

$$
F^{*}=F+\epsilon L
$$

then has the properties described in the last paragraph. Consequently (10.11) holds, that is

$$
\liminf _{q=\infty} k_{q}^{-2}\left[E_{F}^{*}\left(C_{q}\right)+\epsilon E_{L}^{*}\left(C_{q}\right)\right] \geqq 2^{-1} \int_{0}^{1}\left(F_{p^{i} p^{i}}+\epsilon L_{p^{i} p^{i}}\right) \xi_{0}^{i} \xi_{0}^{j} d t .
$$

This equality must hold for all positive values of $\epsilon$. By the use of Lemma 9.3 it is seen that it must also hold when $\epsilon=0$. This proves Lemma 10.1.

11. Completion of the proof of Theorem 4.2. We are now in position to complete the proof of Theorem 4.2. As a first step let

$$
l^{0}=0 \text { or } 1, \quad l^{\sigma}, \quad m^{\beta}(a, y)
$$


be a set of multipliers with which $C_{0}$ satisfies condition $\mathrm{A}$ and let $J(C)$ be the corresponding function (2.12). As in $\$ 7$ we write

$$
J(C)=J^{*}(C)+E_{F}^{*}(C)
$$

and observe that $E_{F}{ }^{*}\left(C_{0}\right)=0$ since $\dot{y}_{0}=p_{0}$. We have accordingly

$$
J\left(C_{q}\right)-J\left(C_{0}\right)=J^{*}\left(C_{q}\right)-J^{*}\left(C_{0}\right)+E_{F}^{*}\left(C_{q}\right) .
$$

Using Lemmas 5.2 and 9.1 it is seen that

(11.3) $\quad \limsup _{q=\infty} \frac{J\left(C_{q}\right)-J\left(C_{0}\right)}{k_{q}^{2}}=2^{-1} J_{2}^{*}\left(\gamma_{0}\right)+\underset{q=\infty}{\limsup } k_{q}^{-2} E_{F}^{*}\left(C_{q}\right) \leqq 0$.

With the help of this relation we shall prove the following lemma.

\section{LEMMA 11.1. The variation $\gamma_{0}$ is not essentially null.}

In order to prove this result suppose that $\gamma_{0}$ is essentially null. Then $\gamma_{0}$ is of the form

$\gamma_{0}$ :

$$
\alpha_{0}^{h}=0, \quad \eta_{0}^{i}(t)=\theta(t) \dot{y}_{0}^{i}(t)
$$$$
(0 \leqq t \leqq 1)
$$

where $\theta(0)=\theta(1)=0$. Since $\left|\dot{y}_{0}\right|=L\left(C_{0}\right)$ we have $\dot{y}_{0} i_{\ddot{y}_{0}}{ }^{i}=0$ and hence

$$
\dot{y}_{0}^{i} \dot{y}_{0}^{i}=\theta \dot{y}_{0}^{i} \ddot{y}_{0}^{i}+\dot{\theta} \dot{y}_{0}^{i} \dot{y}_{0}^{i}=\dot{\theta} L\left(C_{0}\right)^{2} \text {. }
$$

Combining this result with (8.1) it is seen that $\dot{\theta}$ is a constant and hence that $\theta$ is linear in $t$. Since $\theta(0)=\theta(1)=0$ we must have $\theta \equiv 0$. Consequently $\gamma_{0}$ is of the form

$\gamma_{0}$ :

$$
\alpha_{a}^{h}=0, \quad \eta_{0}^{i} \equiv 0
$$$$
(0 \leqq t \leqq 1) \text {. }
$$

It follows that the quantity $J_{2}^{*}\left(\gamma_{0}\right)$ appearing in (11.3) is zero and since $E_{F}^{*}\left(C_{q}\right) \geqq 0$ for large values of $q$ we must have

$$
\lim _{q=\infty} k_{q}^{-2} E_{F}^{*}\left(C_{q}\right)=0 \text {. }
$$

Since we can suppose, by virtue of condition $\mathrm{A}^{\prime}$ for $C_{0}$, that the multipliers (11.1) have been chosen so that $F(a, y, p) E$-dominates $L(p)=|p|$, it follows that there is a constant $b>0$ such that

$$
E_{L}^{*}\left(C_{q}\right) \leqq b E_{F}^{*}\left(C_{q}\right)
$$

for large values of $q$ and hence that

$$
\lim k_{q}^{-2} E_{L}^{*}\left(C_{q}\right)=0 .
$$

This result also could be obtained from the inequality (9.17). Using the formula (8.3) it is seen that 


$$
\lim _{q=\infty} \frac{L\left(C_{q}\right)-L\left(C_{0}\right)}{k_{q}}=0 .
$$

Consider now the identity

$$
\left|\dot{y}_{q}-p_{q}\right|^{2}=\left|L\left(C_{q}\right)-L\left(C_{0}\right)\right|^{2}+2 L\left(C_{0}\right) E_{L}\left(p_{q}, \dot{y}_{q}\right)
$$

which follows from the relations $\left|\dot{y}_{q}\right|=L\left(C_{q}\right),\left|p_{q}\right|=L\left(C_{0}\right)$, and the formula

$$
|p| E_{L}(p, \dot{y})=|\dot{y}||p|-\dot{y}^{i} p^{i} \text {. }
$$

If the terms of this identity are divided by $k_{q}{ }^{2}$, then in view of the relations (11.4), (11.5) and

$$
\dot{y}_{0}=p_{0}, \quad \dot{y}_{q}-p_{q}=k_{q}\left(\dot{\eta}_{q}-\pi_{q}\right)
$$

it is found that

But

$$
\lim _{Q=\infty} \int_{0}^{1}\left|\dot{\eta}_{q}-\pi_{q}\right|^{2} d t=0
$$

$$
\int_{0}^{1}\left|\dot{\eta}_{Q}-\pi_{q}\right|^{2} d t=\int_{0}^{1}\left|\dot{\eta}_{q}\right|^{2} d t-2 \int_{0}^{1} \pi_{q}^{i}\left(\dot{\eta}_{q}^{i}-\pi_{q}^{i}\right) d t-\int_{0}^{1}\left|\pi_{q}\right|^{2} d t
$$

Hence using (6.5) and (6.9) we have

$$
\lim _{q=\infty} \int_{0}^{1}\left|\dot{\eta}_{q}\right|^{2}=0
$$

On the other hand by virtue of (5.9) and (5.10) with $\alpha_{0}{ }^{h}=\eta_{0}{ }^{i}=0$ it is seen that

$$
\lim _{q=\infty} \int_{0}^{1}\left|\dot{\eta}_{q}\right|^{2}=1 \text {. }
$$

In view of this contradiction $\gamma_{0}$ cannot be essentially null, as was to be proved.

LEMMA 11.2. If $C_{0}$ satisfies condition A with the multipliers (11.1) and $J_{2}(\gamma)$ is the second variation of the function $J(C)$ determined by these multipliers, then the inequality $J_{2}\left(\gamma_{0}\right) \leqq 0$ holds.

For by the use of Lemmas 9.1 and 10.1 and the formulas (11.2) and

$$
J_{2}\left(\gamma_{0}\right)=J_{2}^{*}\left(\gamma_{0}\right)+\int_{0}^{1} F_{p^{i} p^{i}}\left(\dot{\eta}_{0}^{i}-\pi_{0}^{i}\right)\left(\dot{\eta}_{0}^{j}-\pi_{0}^{j}\right) d t
$$

it is seen that

$$
\liminf _{q=\infty} \frac{J\left(C_{q}\right)-J\left(C_{0}\right)}{k_{q}^{2}} \geqq 2^{-1} J_{2}\left(\gamma_{0}\right) .
$$

In view of (11.3) we have accordingly $J_{2}\left(\gamma_{0}\right) \leqq 0$, as was to be proved. 
By virtue of Lemmas 8.1, 11.1 and 11.2 it is seen that the variation $\gamma_{0}$ has the properties described in Theorem 4.2. This theorem is therefore established.

THEOREM 11.1. Suppose that for every admissible variation $\gamma$, that is not essentially null, there exists a set of multipliers $l^{0}=0, l^{\sigma}, m^{\beta}(a, y)$ with which $C_{0}$ satisfies condition $\mathrm{A}^{\prime}$ and for which the inequality $J_{2}(\gamma)>0$ holds, where $J_{2}(\gamma)$ is given by (2.18). Then there is a neighborhood $\mathfrak{F}$ of $C_{0}$ in ay-space containing no admissible arc $C \neq C_{0}$.

It should be observed that the hypotheses of this theorem are satisfied if we replace $f(a, y, p)$ and $g(a)$ by $f \equiv 0$ and $g \equiv 0$, respectively. This is because $f$ and $g$ enter the hypotheses only in the combination $l^{\circ} f, l^{\circ} \mathrm{g}$ and $l^{\circ}$ is zero anyhow. After this replacement we can assume that $l^{\circ}=1$. Then by Theorem 4.1 we can select a constant $\epsilon>0$ and a neighborhood $\mathfrak{F}$ of $C_{0}$ in $a y$-space such that

$$
0=I(C)-I\left(C_{0}\right) \geqq \min \left|\epsilon, \epsilon K\left(C, C_{0}\right)\right| .
$$

Since $K\left(C, C_{0}\right)$ is not negative this implies that it is zero and hence that $C$ is identical with $C_{0}$.

12. Sufficient conditions for a weak relative minimum. A theorem analogous to Theorem 4.1 holds for a weak relative minimum. The $\operatorname{arc} C_{0}$ will be said to satisfy the condition $A_{0}$ with a set of multipliers

$$
l^{0} \geqq 0, \quad l^{\sigma}, \quad m^{\beta}(a, y)
$$

if the following conditions hold: the functions $m^{\beta}(a, y)$ are of class $C^{\prime}$; the Euler-Lagrange equations (2.14) and the transversality conditions (2.15) are satisfied along $C_{0}$, the function $F(a, y, p)$ given by $(2.11)$ is such that

$$
F_{p^{i} p^{i} \pi^{i} \pi^{j} \geqq 0}
$$

holds along $C_{0}$ for all solutions $\pi$ of the equations $\phi_{p}^{\beta} \pi^{i}=0$. If in addition the equality in (12.2) holds only in case $\pi^{i}=\rho \dot{y}_{0}{ }^{i}$, then $C_{0}$ will be said to satisfy the condition $\mathrm{A}_{0}$.

We have the following theorem due essentially to McShane $\left({ }^{11}\right)$.

THEOREM 12.1. Let $C_{0}$ be an admissible arc satisfying the conditions described in \$2 and having associated with it a set of multipliers (12.1) with which it satisfies the conditions $\mathrm{A}_{0}{ }^{\prime}$. If for every admissible variation $\gamma$, not essentially null, there exists a set of multipliers (12.1) with which $C_{0}$ satisfies condition $\mathrm{A}_{0}$ and is such that $J_{2}(\gamma)>0$, where $J_{2}(\gamma)$ is the second variation (2.18) of the corresponding function $J(C)$, then there is a neighborhood $\Re_{0}$ of the elements $(a, y, p)$ on $C_{0}$ and $a$ constant $\epsilon>0$ such that the inequality

$$
I(C)-I\left(C_{0}\right) \geqq \epsilon K\left(C, C_{0}\right)
$$

holds for every admissible arc $C$ in $\Re_{0}$.

(i1) Loc. cit. p. 349. 
Here it is understood that an arc

$C: \quad a, \quad y(t)$ $\left(t^{1} \leqq t \leqq t^{2}\right)$

is in $\Re_{0}$ if the element $[a, y(t), \dot{y}(t)]$ is in $\Re_{0}$ for almost all values of $t$ on $0 \leqq t \leqq 1$.

It should be observed that in Theorem 12.1 no generality is lost if the condition $A_{0}$ is replaced by condition $A_{0}{ }^{\prime}$. This follows because if the multipliers (12.1) are chosen related to $C_{0}$ and a variation $\gamma$, not essentially null, as described in the theorem, and if $\bar{l}^{0} \geqq 0, \bar{l}, \bar{m}(a, y)$ is a set with which $C_{0}$ satisfies condition $\mathrm{A}_{0}{ }^{\prime}$, then for a suitably small constant $b$ the arc $C_{0}$ will satisfy the condition $\mathrm{A}_{0}{ }^{\prime}$ with the multipliers

$$
l^{0}+b \bar{l}^{0}, \quad l^{\sigma}+b \bar{l}^{\sigma}, \quad m^{\beta}(a, y)+b \bar{m}^{\beta}(a, y)
$$

and have $J_{2}(\gamma)>0$ for the corresponding second variation (2.18). Thus condition $\mathrm{A}_{0}{ }^{\prime}$ can be assumed to hold throughout. In view of Theorem 3.1 of the first paper the corresponding function $F(a, y, p, l, m(a, y))$ weakly $E$-dominates $L(p)=|p|, f^{\sigma}, \phi^{\beta}$ near $C_{0}$ on $\mathfrak{D}$. Delete from $\mathfrak{D}$ all the elements $(a, y, p)$ which are not in the neighborhood $\Re_{0}$ occurring in the definition of weak $E$ dominance. On the remaining set, which we again call $\mathfrak{D}$, the functions $L, f^{\circ}$ and $\phi^{\beta}$ are $E$-dominated by $F$ near $C_{0}$. Consequently, by Theorem 4.1 we can diminish $\Re_{0}$, if necessary, so that the inequality

$$
I(C)-I\left(C_{0}\right) \geqq \min \left|\epsilon, \epsilon K\left(C, C_{0}\right)\right|
$$

holds for every admissible arc $C$ in $\Re_{0}, \epsilon$ being a suitably chosen positive constant. If $\Re_{0}$ is taken small enough we have $K\left(C, C_{0}\right)<1$, so that (12.3) holds. This proves Theorem 4.1.

As an analogue of Theorem 4.3 we have the following theorem.

Theorem 12.2. If $C_{0}$ satisfied the hypotheses described in Theorem 12.1, there is a neighborhood $\Re_{0}$ of the elements $(a, y, p)$ on $C_{0}$ such that for every neighborhood $\mathfrak{F}$ of $C_{0}$ in ay-space there is a constant $\rho>0$ such that the inequality

$$
I(C)-I\left(C_{0}\right)>\rho
$$

holds for every admissible arc $C_{0}$ in $\Re_{0}$ having on it a point $(a, y)$ that is not in $\mathfrak{F}$.

For let $\Re_{0}$ and $\epsilon$ be chosen as described in Theorem 12.1. For a given $\mathfrak{F}$ choose $\rho$ so that $2 \rho$ is the greatest lower bound of $\epsilon K\left(C, C_{0}\right)$ for all arcs $C$ in $\Re_{0}$ that are not in $\mathfrak{F}$. Clearly $\rho>0$. The relation (12.4) then follows from (12.3) and the relation $\epsilon K\left(C, C_{0}\right)>\rho$.

This proves Theorem 12.2.

The University of Chicago, Chicago, Ill. 